Z Rheumatol 2013 · 72:422

DOI 10.1007/s00393-012-1126-1

Online publiziert: 8. Juni 2013

๑) Springer-Verlag Berlin Heidelberg 2013

Jürgen Wollenhaupt ${ }^{1} \cdot$ Kristian Reich $^{2}$

${ }^{1}$ Rheumatologikum Hamburg, Schön Klinik Hamburg Eilbeck

${ }^{2}$ Dermatologikum Hamburg

\title{
Haut als Manifestationsorgan entzündlich-rheumatischer Erkrankungen
}

innerhalb dieses Themenschwerpunkts, sondern gesondert in einer späteren Ausgabe der Zeitschrift für Rheumatologie von Sattler dargestellt werden.

Die Haut bei Lupus erythematodes ist Gegenstand des Beitrags von Sticherling, der sowohl die rein kutanen Formen als auch die Hautmanifestationen des systemischen Lupus erläutert. Für den rheumatologischen Praxisalltag relevant sind auch die über die systemischen Therapieansätze hinausgehenden Behandlungshinweise zu UV-Schutz, Einflüssen von Rauchen und Alkohol und topischen Glukokortikoiden. andere besonders markante interdisziplinäre Aspekte, deren Kenntnis im rheumatologischen Alltag wichtig ist.

Sog. paradoxe Hautreaktionen während einer Biological-Therapie mit TNFAntagonisten stellen eine seltene unerwünschte Wirkung dieser Substanzgruppe dar. Die Autoren Weisenseel und Reich beschreiben die Varianten dieser Hautreaktionen und geben praktische Hinweise zu therapeutischen Konsequenzen.

Das Problem Hautmalignome unter Therapie mit Biologicals, das eine eingehende Darstellung verdient, wird nicht

\section{Infobox}

In einer der nächsten Ausgaben der Zeitschrift für Rheumatologie erscheint zu dem Thema Haut als Manifestationsorgan entzündlich-rheumatischer Erkrankungen auch der Beitrag „Kutane Malignome unter Biolocial-Therapie" von Frau Dr. E. Sattler, München.

\section{D) Rheumatologie und Dermatologie sind seit jeher eng miteinander verknüpfte Fachgebiete}

Die Vaskulitiden der Haut stehen im Mittelpunkt des Beitrags von Sunderkötter, der die klinischen Manifestationen der vermeintlich begrenzten kutanen Formen und der systemischen Vaskulitiden darstellt und in die aktuelle ChapelHill-Klassifikation einordnet. Das große Spektrum der von der Livedo racemosa bis zur septischen Vaskulitis reichenden Symptome und ihre oft schwierige Differenzierung verdeutlicht in besonderem Maß, wie wichtig die enge Zusammenarbeit von Dermatologen und Rheumatologen ist.

Mit den im Themenschwerpunkt ausgewählten Beiträgen sind die Gemein- samkeiten von Dermatologie und Rheumatologie bei Weitem nicht erschöpft. Aber auch über weitere klinische Aspekte hinaus stimulieren sich beide Gebiete bei dem wachsenden Verständnis der Immunpathogenese der jeweiligen organbezogenen oder systemischen entzündlichen Erkrankungen und deren immunmodulatorischen Therapieansätzen: Daraus resultiert eine intellektuell spannende und täglich interessante Kooperation.

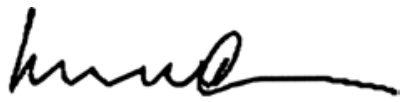

Jürgen Wollenhaupt

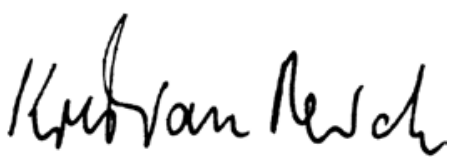

Kristian Reich

\section{Korrespondenzadresse}

Prof. Dr. Jürgen Wollenhaupt Rheumatologikum Hamburg, Schön Klinik Hamburg Eilbeck Dehnhaide 120, 22081 Hamburg wollenhaupt@rheumatologikum.de 